\title{
Global solutions of wave equations with multiple nonlinear source terms under acoustic boundary conditions
}

Shoubo $\mathrm{Jin}^{1 *}$ (D) and Jian $\mathrm{Li}^{2}$

"Correspondence:

jin_shoubo@163.com

1 School of Mathematics and

Statistics, Suzhou University,

Suzhou, 234000, China

Full list of author information is

available at the end of the article

\section{Springer}

\begin{abstract}
Under the acoustic boundary conditions, the initial boundary value problem of a wave equation with multiple nonlinear source terms is considered. This paper gives the energy functional of regular solutions for the wave equation and proves the decreasing property of the energy functional. Firstly, the existence of a global solution for the wave equation is proved by the Faedo-Galerkin method. Then, in order to obtain the nonexistence of global solutions for the wave equation, a new functional is defined. When the initial energy is less than zero, the special properties of the new functional are proved by the method of contraction. Finally, the conditions for the nonexistence of global solutions of the wave equation with acoustic boundary conditions are analyzed by using these special properties.
\end{abstract}

MSC: 35L05; 35L30; 35L35

Keywords: Wave equation; Acoustic boundary conditions; Global solution; Existence; Nonexistence

\section{Introduction}

Wave equation is a partial differential equation describing wave phenomenon, it can be used in many different fields, such as acoustics, electromagnetics, fluid mechanics, and so forth. The existence of global solutions has been one of the key issues in the study of wave equation. As we all know, there are three kinds of boundary conditions commonly used in wave equation, namely Dirichlet condition, Neumann condition, and mixed boundary condition; however, the three conditions signify that the waves travel in the same medium. In fact, when the sound waves enter from one medium to another, some of the sound waves will be reflected back and some of them will be transmitted through, so it is necessary to discuss the wave equation under the acoustic boundary conditions. In this paper, we analyze the existence and blow-up of the global solution of the wave equation under the acoustic boundary conditions. We consider the following wave equation with acoustic boundary conditions:

$$
u_{t t}-\alpha \operatorname{div}\left(|\nabla u|^{m-2} \nabla u\right)-\operatorname{div}\left(\left|\nabla u_{t}\right|^{\beta-2} \nabla u_{t}\right)-\gamma \Delta u_{t}
$$

(c) The Author(s) 2021. This article is licensed under a Creative Commons Attribution 4.0 International License, which permits use sharing, adaptation, distribution and reproduction in any medium or format, as long as you give appropriate credit to the original author(s) and the source, provide a link to the Creative Commons licence, and indicate if changes were made. The images or other third party material in this article are included in the article's Creative Commons licence, unless indicated otherwise in a credit line to the material. If material is not included in the article's Creative Commons licence and your intended use is not permitted by statutory regulation or exceeds the permitted use, you will need to obtain permission directly from the copyright holder. To view a copy of this licence, visit http://creativecommons.org/licenses/by/4.0/. 


$$
=\sum_{i=1}^{l_{1}} a_{i}|u|^{p_{i}-2} u-\sum_{j=1}^{l_{2}} b_{j}|u|^{q_{j}-2} u, \quad x \in \Omega, t>0,
$$

the initial boundary conditions are as follows:

$$
\left\{\begin{array}{l}
\left.u\right|_{\Gamma_{0} \times(0,+\infty)}=0 \\
\gamma \frac{\partial u_{t}}{\partial n}+\alpha|\nabla u|^{m-2} \frac{\partial u}{\partial n}+\left|\nabla u_{t}\right|^{\beta-2} \frac{\partial u_{t}}{\partial n}=h(x) y_{t}, \quad(x, t) \in \Gamma_{1} \times(0,+\infty) \\
u_{t}+f(x) y_{t}+q(x) y=0, \quad(x, t) \in \Gamma_{1} \times(0,+\infty) \\
u(x, 0)=u_{0}(x), \quad u_{t}(x, 0)=u_{1}(x), \quad x \in \Omega \\
y(x, 0)=y_{0}(x), \quad x \in \Gamma_{1}
\end{array}\right.
$$

where $\alpha, \gamma, a_{i}, b_{j}>0,2<\beta<m$, and $2<q_{l_{2}}<\cdots<q_{2}<q_{1}<p_{1}<p_{2}<\cdots<p_{l_{1}}<r^{*}$ are constants, $r^{*}=\frac{n m}{n-m}$ is the critical Sobolev index of the space $W^{1, m}(\Omega) . \Omega \subset R^{n}(n \geq 3)$ is a bounded domain with the smooth boundary $\Gamma=\Gamma_{0} \cup \Gamma_{1}$, where $\Gamma_{0}$ and $\Gamma_{1}$ are disjoint and closed. The unit outward normal vector to the boundary is denoted by $\frac{\partial u}{\partial n} \cdot f, q, h: \Gamma_{1} \rightarrow R^{+}$ are bounded essential functions, satisfying $0<q_{0} \leq q(x)$ and $0<h_{0} \leq h(x)$ for any $x \in \Gamma_{1}$. The differentiable function $y$ indicates the material of the surface.

The second and third equations in (2) are known as acoustic boundary conditions, and they simulate a porous boundary. For decades, many achievements have been achieved on the wave equations with Dirichlet conditions, Neumann condition, or mixed boundary condition; however, these conditions cannot perfectly deal with the problem of wave propagation in mixed media. Hence, acoustic boundary conditions with intuitive models began to be widely considered by scholars. Morse and Ingard [1] introduced acoustic boundary conditions in 1968. Beale and Rosencrans [2] firstly proposed a general form of acoustic boundary conditions and proved the existence and regularity of the solution of wave equation under acoustic boundary conditions. Recently, many results have been obtained under acoustic boundary conditions. For instance, Gerbi and Sai-Houari studied a class of semi-linear wave equations and proved the existence of local solutions by the Faedo-Galerkin approximation and contraction mapping theorem in [3]. Frigeri analyzed the global asymptotic behavior of solutions of a class of wave equations in [4]. The decay rate of viscoelastic wave equations are discussed in [5]. The existence and nonexistence of global solutions for viscoelastic wave equations are analyzed in [6]. Nonlinear and quasi-linear viscoelastic wave equations are studied in [7] and [8], respectively. Jeong et al. obtained the nonexistence conditions of global solutions for a class of quasi-linear wave equations with acoustic boundary conditions by using the energy functional method in [9]. The global solutions of wave equations with time delay are analyzed in [10]. More studies on the wave equation with acoustic boundary conditions can be found in [11-13].

For the wave equation with positive definite energy $u_{t t}-\Delta u=-|u|^{p-2} u$, the existence of solutions can be proved by the Galerkin approximation method. For the wave equation with nonpositive definite energy $u_{t t}-\Delta u=|u|^{p-2} u$, the global existence of solutions was proved by using potential well method in 1968 [14], and the existence, blowup behavior, and vacuum isolation of solutions were also discussed in [15] and [16]. In 2007, [17] and [18] introduced a class of wave equations with different sign source terms, $u_{t t}-\Delta u=a|u|^{p-2} u-b|u|^{q-2} u$, and investigated the existence and blow-up of global solutions. Recently, [19] proposed a class of wave equations with multiple nonlinear source 
terms and studied the existence and nonexistence of global solutions. [20] and [21] applied multiple nonlinear source terms to Schrodinger equation and Boussinesq equation, and proved the existence and blow-up of global solutions. Wave equations with multiple nonlinear source terms are also investigated in [22] and [23]. The nonlinear wave equation with weak and strong damping terms and logarithmic source term is discussed in [24]. However, the mentioned wave equations with multiple nonlinear source terms are under Dirichlet condition, Neumann condition, or mixed boundary condition, and there are few results under the acoustic boundary conditions. In this paper, the existence and nonexistence of global solutions of Eq. (1) are considered under acoustic boundary conditions, the method used in this paper is partly from that in [9] and [25].

Since Eqs. (1)-(2) include multiple nonlinear source terms and acoustic boundary conditions, the potential well theory in [17-23] cannot be used directly. In addition, $m$ and $\beta$ order Laplacian term makes the problem more complicated. The difficulty of this paper is to estimate $\left\|u_{t t}\right\|_{2}$ and $\|u(t)\|_{p_{i}}$, we need to deduce more precisely by using Minkowski's inequality, Hölder's inequality, and Young's inequality. Finally, the existence and nonexistence of the global solutions of the equation are obtained.

\section{Existence theorem}

Many results have been obtained on the existence of global solutions for wave equations with acoustic boundary conditions $[3,5,7,8,12]$, the Faedo-Galerkin method is one of the most used methods to prove the existence of solutions. [25] and [26] respectively proved the existence of solutions for two different kinds of wave equations with acoustic boundary conditions by using the Faedo-Galerkin method, but these two kinds of equations contain neither multiple nonlinear source terms nor $m$ and $\beta$ order Laplacian operators. In this paper, we use the Faedo-Galerkin method to prove the existence of global solutions of Eqs. (1)-(2).

Some notations are given as follows:

$$
\begin{aligned}
& W_{\Gamma_{0}}^{1, s}(\Omega)=\left\{u \in W^{1, s}(\Omega)|u|_{\Gamma_{0}}=0\right\}, \quad(u, v)=\int_{\Omega} u v d x, \\
& (u, v)_{\Gamma_{1}}=\int_{\Gamma_{1}} u v d \Gamma, \quad\|v\|_{p}=\left(\int_{\Omega}|u|^{p} d x\right)^{\frac{1}{p}} .
\end{aligned}
$$

The energy functional corresponding to Eqs. (1)-(2) is

$$
\begin{aligned}
E(t)= & \frac{1}{2} \int_{\Omega} u_{t}^{2} d x+\frac{1}{m} \alpha \int_{\Omega}|\nabla u|^{m} d x-\sum_{i=1}^{l_{1}} \frac{a_{i}}{p_{i}} \int_{\Omega}|u|^{p_{i}} d x \\
& +\sum_{j=1}^{l_{2}} \frac{b_{j}}{q_{j}} \int_{\Omega}|u|^{q_{j}} d x+\frac{1}{2} \int_{\Gamma_{1}} h(x) q(x) y^{2}(t) d \Gamma .
\end{aligned}
$$

For the convenience of calculation, the letter $C$ in different formulas stands for a different positive constant. We first give the monotonicity theorem of energy functional as follows.

Lemma 1 If $u$ is a regular solution of Eq. (1)-(2), then the energy functional satisfies

$$
E^{\prime}(t)=-\gamma \int_{\Omega}\left|\nabla u_{t}\right|^{2} d x-\int_{\Omega}\left|\nabla u_{t}\right|^{\beta} d x-\int_{\Gamma_{1}} h(x) f(x) y_{t}^{2}(t) d \Gamma \leq 0
$$


and

$$
E(t) \leq E(0), \quad(t \geq 0)
$$

Proof Multiplying Eq. (1) by $u_{t}$ and integrating on $\Omega$, we can get the conclusion from the initial boundary conditions.

Remark 1 Formula (5) represents the energy inequality of the wave equation. It can be seen from formula (4) that the energy is nonincreasing with respect to $t$. The proof of the energy inequality of the wave equation with acoustic boundary conditions is similar to that in $[6,10,27]$, and [28]. In this lemma, the energy functional has a more complicated form than in the previous literature works.

Theory 1 Suppose that the initial values of Eqs. (1)-(2) satisfies $u_{0}(x) \in W_{\Gamma_{0}}^{1, m}(\Omega)$ and $u_{1}(x) \in W_{\Gamma_{0}}^{1, \beta}(\Omega)$, if $p_{l_{1}} \leq \beta$, then there exists a set of global solutions $(u(x, t), y(x, t))$, satisfying

$$
\begin{aligned}
& u \in L^{\infty}\left(0, T ; W^{1, m}(\Omega) \cap L^{q l_{2}}(\Omega)\right), \quad u_{t} \in L^{\infty}\left(0, T ; W^{1, \beta}(\Omega)\right), \\
& u_{t t} \in L^{\infty}\left(0, T ; L^{2}(\Omega)\right)
\end{aligned}
$$

and $y, y_{t}, y_{t t} \in L^{\infty}\left(0, T ; L^{2}\left(\Gamma_{1}\right)\right)$ for any $T>0$.

Proof Let $\left\{w_{s}\right\}$ and $\left\{\xi_{s}\right\}$ be the basic functions of the Sobolev space $W_{\Gamma_{0}}^{1, m}(\Omega)$. The approximate solutions of Eqs. (1)-(2) are constructed by the Faedo-Galerkin method as follows:

$$
u_{l \varepsilon}(x, t)=\sum_{s=1}^{l} d_{l}^{s}(t) w_{s}(x), \quad y_{l \varepsilon}(x, t)=\sum_{s=1}^{l} b_{l}^{s}(t) \xi_{s}(x)
$$

and

$$
\left\{\begin{array}{l}
\left(u_{l \varepsilon}^{\prime \prime}, w_{s}\right)+\alpha\left(\left|\nabla u_{l \varepsilon}\right|^{m-2} \nabla u_{l \varepsilon}, \nabla w_{s}\right)+\left(\left|\nabla u_{l \varepsilon}^{\prime}\right|^{\beta-2} \nabla u_{l \varepsilon}^{\prime}, \nabla w_{s}\right)+\gamma\left(\nabla u_{l \varepsilon}^{\prime}, \nabla w_{s}\right) \\
\quad-\left(h(x) y_{l \varepsilon}^{\prime}, w_{s}\right)_{\Gamma_{1}}=\sum_{i=1}^{l_{1}} a_{i}\left(\left|u_{l \varepsilon}\right|^{p_{i}-2} u_{l \varepsilon}, w_{s}\right)-\sum_{j=1}^{l_{2}} b_{j}\left(\left|u_{l \varepsilon}\right|^{q_{j}-2} u_{l \varepsilon}, w_{s}\right), \\
\varepsilon\left(y_{l \varepsilon}^{\prime \prime}, \xi_{s}\right)_{\Gamma_{1}}+\left(h(x)\left[u_{l \varepsilon}^{\prime}+f(x) y_{l \varepsilon}^{\prime}+q(x) y_{l \varepsilon}\right], \xi_{s}\right)_{\Gamma_{1}}=0, \\
u_{l \varepsilon}(0)=\sum_{s=1}^{l}\left(u_{0}, w_{s}\right) w_{s}, \quad u_{l \varepsilon}^{\prime}(0)=\sum_{s=1}^{l}\left(u_{1}, w_{s}\right) w_{s}, \\
y_{l \varepsilon}(0)=-\frac{\left.u_{l \varepsilon}^{\prime}(0)+f(x)\right)_{l \varepsilon}^{\prime}(0)}{q(x)}, \\
y_{l \varepsilon}^{\prime}(0)=\frac{\gamma \frac{\partial u_{l \varepsilon}^{\prime}(0)}{\partial n}+\alpha\left|\nabla u_{l \varepsilon}(0)\right|^{m-2} \frac{\partial u_{l \varepsilon}(0)}{\partial n}+\left|\nabla u_{l \varepsilon}^{\prime}(0)\right|^{\beta-2} \frac{\partial u_{\varepsilon}^{\prime}(0)}{\partial n}}{h(x)} .
\end{array}\right.
$$

(1) First step estimation.

Multiplying (7) by the functions $d_{l}^{\prime s}(t)$ and $b_{l}^{\prime s}(t)$, respectively, and summing with respect to $s$, we have

$$
\begin{aligned}
& \frac{d}{d t}\left(\frac{1}{2} \int_{\Omega} u_{l \varepsilon}^{\prime 2} d x+\frac{\alpha}{m} \int_{\Omega}\left|\nabla u_{l \varepsilon}\right|^{m} d x-\sum_{i=1}^{l_{1}} \frac{a_{i}}{p_{i}} \int_{\Omega}\left|u_{l \varepsilon}\right|^{p_{i}} d x+\sum_{j=1}^{l_{2}} \frac{b_{j}}{q_{j}} \int_{\Omega}\left|u_{l \varepsilon}\right|^{q_{j}} d x\right. \\
& \left.\quad+\frac{1}{2} \varepsilon \int_{\Gamma_{1}} y_{l \varepsilon}^{\prime 2} d \Gamma+\frac{1}{2} \int_{\Gamma_{1}} h q y_{l \varepsilon}^{2} d \Gamma\right)+\int_{\Gamma_{1}} h f y_{l \varepsilon}^{\prime 2} d \Gamma
\end{aligned}
$$




$$
=-\gamma \int_{\Omega}\left|\nabla u_{l \varepsilon}^{\prime}\right|^{2} d x-\int_{\Omega}\left|\nabla u_{l \varepsilon}^{\prime}\right|^{\beta} d x .
$$

Integrating from 0 to $t$, we get

$$
\begin{aligned}
\frac{1}{2} \int_{\Omega} u_{l \varepsilon}^{\prime 2} d x & +\frac{\alpha}{m} \int_{\Omega}\left|\nabla u_{l \varepsilon}\right|^{m} d x-\sum_{i=1}^{l_{1}} \frac{a_{i}}{p_{i}} \int_{\Omega}\left|u_{l \varepsilon}\right|^{p_{i}} d x \\
& +\sum_{j=1}^{l_{2}} \frac{b_{j}}{q_{j}} \int_{\Omega}\left|u_{l \varepsilon}\right|^{q_{j}} d x+\frac{1}{2} \varepsilon \int_{\Gamma_{1}} y_{l \varepsilon}^{\prime 2} d \Gamma \\
& +\frac{1}{2} \int_{\Gamma_{1}} h q y_{l \varepsilon}^{2} d \Gamma+\int_{0}^{t} \int_{\Gamma_{1}} h f y_{l \varepsilon}^{\prime 2} d \Gamma d \tau \\
& +\gamma \int_{0}^{t} \int_{\Omega}\left|\nabla u_{l \varepsilon}\right|^{\prime 2} d x d \tau+\int_{0}^{t} \int_{\Omega}\left|\nabla u_{l \varepsilon}^{\prime}\right|^{\beta} d x d \tau \\
= & \frac{1}{2} \int_{\Omega} u_{l \varepsilon}^{\prime 2}(0) d x+\frac{\alpha}{m} \int_{\Omega}\left|\nabla u_{l \varepsilon}(0)\right|^{m} d x-\sum_{i=1}^{l_{1}} \frac{a_{i}}{p_{i}} \int_{\Omega}\left|u_{l \varepsilon}(0)\right|^{p_{i}} d x \\
& +\sum_{j=1}^{l_{2}} \frac{b_{j}}{q_{j}} \int_{\Omega}\left|u_{l \varepsilon}(0)\right|^{q_{j}} d x \\
& +\frac{1}{2} \varepsilon \int_{\Gamma_{1}} y_{l \varepsilon}^{\prime 2}(0) d \Gamma+\frac{1}{2} \int_{\Gamma_{1}} h q y_{l \varepsilon}^{2}(0) d \Gamma .
\end{aligned}
$$

Let us estimate the functional $\sum_{i=1}^{l_{1}} \frac{a_{i}}{p_{i}} \int_{\Omega}\left|u_{l \varepsilon}\right|^{p_{i}} d x$ by use of the method in [9] and [22]. The following formula can be obtained by simple calculation:

$$
u_{l \varepsilon}(t)=u_{l \varepsilon}(0)+\int_{0}^{t} u_{l \varepsilon}^{\prime}(\tau) d \tau
$$

Using Minkowski's inequality and Hölder's inequality, it holds

$$
\begin{aligned}
\left\|u_{l \varepsilon}(t)\right\|_{p_{i}} & \leq\left\|u_{l \varepsilon}(0)\right\|_{p_{i}}+\int_{0}^{t}\left\|u_{l \varepsilon}^{\prime}(\tau)\right\|_{p_{i}} d \tau \\
\left\|u_{l \varepsilon}(t)\right\|_{p_{i}}^{p_{i}} & \leq 2^{p_{i}}\left[\left\|u_{l \varepsilon}(0)\right\|_{p_{i}}^{p_{i}}+\left(\int_{0}^{t}\left\|u_{l \varepsilon}^{\prime}(\tau)\right\|_{p_{i}} d \tau\right)^{p_{i}}\right] \\
& \leq 2^{p_{i}}\left[\left\|u_{l \varepsilon}(0)\right\|_{p_{i}}^{p_{i}}+t^{p_{i}-1} \int_{0}^{t}\left\|u_{l \varepsilon}^{\prime}(\tau)\right\|_{p_{i}}^{p_{i}} d \tau\right]
\end{aligned}
$$

The following inequality can be obtained by use of Young's inequality and the known condition $p_{i}<\beta$ :

$$
t^{p_{i}-1}\left|u_{l \varepsilon}^{\prime}\right|^{p_{i}} \leq \kappa \frac{p_{i}}{\beta}\left|u_{l \varepsilon}^{\prime}\right|^{\beta}+C_{1 i}(\kappa) \frac{\beta-p_{i}}{\beta} t^{\frac{\beta\left(p_{i}-1\right)}{\beta-p_{i}}}
$$


for any $\kappa>0$, where $C_{1 i}(\kappa)=\kappa^{\frac{p_{i}}{p_{i}-\beta}}$. Then, by using Sobolev's embedding inequality, we can get

$$
\begin{aligned}
t^{p_{i}-1} \int_{0}^{t}\left\|u_{l \varepsilon}^{\prime}(\tau)\right\|_{p_{i}}^{p_{i}} d \tau & \leq \kappa \frac{p_{i}}{\beta} \int_{0}^{t}\left\|u_{l \varepsilon}^{\prime}\right\|_{\beta}^{\beta} d \tau+C_{1 i}(\kappa) \frac{\beta-p_{i}}{\beta} t^{\frac{\beta\left(p_{i}-1\right)}{\beta-p_{i}}+1}|\Omega| \\
& \leq \kappa \frac{p_{i}}{\beta} C_{*} \int_{0}^{t}\left\|\nabla u_{l \varepsilon}^{\prime}\right\|_{\beta}^{\beta} d \tau+C_{1 i}(\kappa) \frac{\beta-p_{i}}{\beta} t^{\frac{p_{i}(\beta-1)}{\beta-p_{i}}}|\Omega|,
\end{aligned}
$$

where $C_{*}$ is the Sobolev embedding constant. Hence, we have

$$
\begin{aligned}
\sum_{i=1}^{l_{1}} \frac{a_{i}}{p_{i}} \int_{\Omega}\left|u_{l \varepsilon}\right|^{p_{i}} d x \leq & \sum_{i=1}^{l_{1}} \frac{a_{i}}{p_{i}} 2^{p_{i}}\left\|u_{l \varepsilon}(0)\right\|_{p_{i}}^{p_{i}}+\sum_{i=1}^{l_{1}} \kappa \frac{a_{i}}{\beta} C_{*} 2^{p_{i}} \int_{0}^{t}\left\|\nabla u_{l \varepsilon}^{\prime}\right\|_{\beta}^{\beta} d \tau \\
& +\sum_{i=1}^{l_{1}} C_{1 i}(\kappa) a_{i} \frac{\beta-p_{i}}{\beta p_{i}} t^{\frac{p_{i}(\beta-1)}{\beta-p_{i}}} 2^{p_{i}}|\Omega| .
\end{aligned}
$$

We can select the appropriate value $\kappa$ to make $\sum_{i=1}^{l_{1}} \kappa \frac{a_{i}}{\beta} C_{*} 2^{p_{i}}=\frac{1}{2}$ and substitute (10) into (8), then there exists a constant $M_{1}(T)>0$ such that

$$
\begin{aligned}
& \frac{1}{2} \int_{\Omega} u_{l \varepsilon}^{\prime 2} d x+\frac{\alpha}{m} \int_{\Omega}\left|\nabla u_{l \varepsilon}\right|^{m} d x+\sum_{j=1}^{l_{2}} \frac{b_{j}}{q_{j}} \int_{\Omega}\left|u_{l \varepsilon}\right|^{q_{j}} d x+\frac{1}{2} \varepsilon \int_{\Gamma_{1}} y_{l \varepsilon}^{\prime 2} d \Gamma+\frac{1}{2} \int_{\Gamma_{1}} h q y_{l \varepsilon}^{2} d \Gamma \\
& \quad+\int_{0}^{t} \int_{\Gamma_{1}} h f y_{l \varepsilon}^{\prime 2} d \Gamma d \tau+\gamma \int_{0}^{t} \int_{\Omega}\left|\nabla u_{l \varepsilon}^{\prime}\right|^{2} d x d \tau+\frac{1}{2} \int_{0}^{t} \int_{\Omega}\left|\nabla u_{l \varepsilon}^{\prime}\right|^{\beta} d x d \tau \\
& \leq M_{1}(T) .
\end{aligned}
$$

(2) Second step estimation.

We firstly estimate $u_{l \varepsilon}^{\prime \prime}(0)$ and $y_{l \varepsilon}^{\prime \prime}(0)$. Taking $t=0, w=u_{l \varepsilon}^{\prime \prime}(0)$, and $\xi=y_{l \varepsilon}^{\prime \prime}(0)$ in Eq. (7), we obtain

$$
\begin{aligned}
\left\|u_{l \varepsilon}^{\prime \prime}(0)\right\|_{2}^{2}= & \alpha\left(\operatorname{div}\left(\left|\nabla u_{l \varepsilon}(0)\right|^{m-2} \nabla u_{l \varepsilon}(0)\right), u_{l \varepsilon}^{\prime \prime}(0)\right)+\left(\operatorname{div}\left(\left|\nabla u_{l \varepsilon}^{\prime}(0)\right|^{\beta-2} \nabla u_{l \varepsilon}^{\prime}(0)\right), u_{l \varepsilon}^{\prime \prime}(0)\right) \\
& +\gamma\left(\Delta u_{l \varepsilon}^{\prime}(0), u_{l \varepsilon}^{\prime \prime}(0)\right)+\sum_{i=1}^{l_{1}} a_{i}\left(\left|u_{l \varepsilon}(0)\right|^{p_{i}-2} u_{l \varepsilon}(0), u_{l \varepsilon}^{\prime \prime}(0)\right) \\
& -\sum_{j=1}^{l_{2}} b_{j}\left(\left|u_{l \varepsilon}(0)\right|^{q_{j}-2} u_{l \varepsilon}(0), u_{l \varepsilon}^{\prime \prime}(0)\right)
\end{aligned}
$$

and $\varepsilon\left\|y_{l \varepsilon}^{\prime \prime}(0)\right\|_{2, \Gamma_{1}}^{2}+\left(h(x)\left[u_{l \varepsilon}^{\prime}(0)+f(x) y_{l \varepsilon}^{\prime}(0)+q(x) y_{l \varepsilon}(0)\right], y_{l \varepsilon}^{\prime \prime}(0)\right)_{\Gamma_{1}}=0$.

Using the known conditions and Hölder's inequality, it follows that there exists a constant $C>0$ such that $\left\|u_{l \varepsilon}^{\prime \prime}(0)\right\|_{2} \leq C$ and $\left\|y_{l \varepsilon}^{\prime \prime}(0)\right\|_{2, \Gamma_{1}}^{2}=0$.

Differentiating Eq. (7) with respect to $t$ and replacing $w_{s}$ and $\xi_{s}$ with $u_{l \varepsilon}^{\prime \prime}(t)$ and $y_{l \varepsilon}^{\prime \prime}(t)$, respectively, it holds

$$
\frac{d}{d t}\left[\frac{1}{2} \int_{\Omega} u_{l \varepsilon}^{\prime \prime 2} d x+\frac{\varepsilon}{2} \int_{\Gamma_{1}} y_{l \varepsilon}^{\prime \prime 2} d \Gamma+\frac{1}{2} \int_{\Gamma_{1}} h q y_{l \varepsilon}^{\prime 2} d \Gamma\right]+\int_{\Omega}\left|\nabla u_{l \varepsilon}^{\prime}\right|^{\beta-2}\left|\nabla u_{l \varepsilon}\right|^{\prime \prime 2} d x
$$


Jun and Li Boundary Value Problems

(2021) 2021:59

Page 7 of 15

$$
\begin{aligned}
& +(\beta-2) \int_{\Omega}\left|\nabla u_{l \varepsilon}^{\prime}\right|^{\beta-4}\left(\nabla u_{l \varepsilon}^{\prime} \cdot \nabla u_{l \varepsilon}^{\prime \prime}\right)^{2} d x+\gamma \int_{\Omega}\left|\nabla u_{l \varepsilon}^{\prime \prime}\right|^{2} d x+\int_{\Gamma_{1}} h f y_{l \varepsilon}^{\prime \prime 2} d \Gamma \\
= & -\alpha \int_{\Omega}\left|\nabla u_{l \varepsilon}\right|^{m-2} \nabla u_{l \varepsilon}^{\prime} \cdot \nabla u_{l \varepsilon}^{\prime \prime} d x \\
& -\alpha(m-2) \int_{\Omega}\left|\nabla u_{l \varepsilon}\right|^{m-4}\left(\nabla u_{l \varepsilon} \cdot \nabla u_{l \varepsilon}^{\prime}\right)\left(\nabla u_{l \varepsilon} \cdot \nabla u_{l \varepsilon}^{\prime \prime}\right) d x \\
& +\sum_{i=1}^{l_{1}} a_{i}\left(p_{i}-1\right) \int_{\Omega}\left|u_{l \varepsilon}\right|^{p_{i}-2} u_{l \varepsilon}^{\prime} u_{l \varepsilon}^{\prime \prime} d x-\sum_{j=1}^{l_{2}} b_{j}\left(q_{j}-1\right) \int_{\Omega}\left|u_{l \varepsilon}\right|^{q_{j}-2} u_{l \varepsilon}^{\prime} u_{l \varepsilon}^{\prime \prime} d x .
\end{aligned}
$$

Using Hölder's inequality and Young's inequality, we have

$$
\begin{aligned}
& \left.\left|-\alpha \int_{\Omega}\right| \nabla u_{l \varepsilon}\right|^{m-2} \nabla u_{l \varepsilon}^{\prime} \cdot \nabla u_{l \varepsilon}^{\prime \prime} d x \\
& \quad-\alpha(m-2) \int_{\Omega}\left|\nabla u_{l \varepsilon}\right|^{m-4}\left(\nabla u_{l \varepsilon} \cdot \nabla u_{l \varepsilon}^{\prime}\right)\left(\nabla u_{l \varepsilon} \cdot \nabla u_{l \varepsilon}^{\prime \prime}\right) d x \mid \\
& \leq\left.\left|\alpha(m-1) \int_{\Omega}\right| \nabla u_{l \varepsilon}\right|^{m-2} \nabla u_{l \varepsilon}^{\prime} \nabla u_{l \varepsilon}^{\prime \prime} d x \mid \\
& \leq \alpha(m-1) \int_{\Omega}\left|\nabla u_{l \varepsilon}\right|^{m-2}\left|\nabla u_{l \varepsilon}^{\prime} \nabla u_{l \varepsilon}^{\prime \prime}\right| d x \\
& \leq \alpha(m-1)\left(\int_{\Omega}\left(\left|\nabla u_{l \varepsilon}\right|^{m-2}\left|\nabla u_{l \varepsilon}^{\prime \prime}\right|\right)^{2} d x\right)^{\frac{1}{2}}\left(\int_{\Omega}\left|\nabla u_{l \varepsilon}^{\prime}\right|^{2} d x\right)^{\frac{1}{2}} \\
& \leq \alpha(m-1) \kappa\left(\int_{\Omega}\left(\left|\nabla u_{l \varepsilon}\right|^{m-2}\left|\nabla u_{l \varepsilon}^{\prime \prime}\right|\right)^{2} d x\right)+\alpha(m-1) C_{1}(\kappa) \int_{\Omega}\left|\nabla u_{l \varepsilon}^{\prime}\right|^{2} d x
\end{aligned}
$$

and

$$
\begin{aligned}
& \left.\left|\sum_{i=1}^{l_{1}} a_{i}\left(p_{i}-1\right) \int_{\Omega}\right| u_{l \varepsilon}\right|^{p_{i}-2} u_{l \varepsilon}^{\prime} u_{l \varepsilon}^{\prime \prime} d x \mid \\
& \quad \leq \sum_{i=1}^{l_{1}} a_{i}\left(p_{i}-1\right) \kappa \int_{\Omega}\left(\left|u_{l \varepsilon}\right|^{p_{i}-2} u_{l \varepsilon}^{\prime \prime}\right)^{2} d x+\sum_{i=1}^{l_{1}} a_{i}\left(p_{i}-1\right) C_{1}(\kappa) \int_{\Omega} u_{l \varepsilon}^{\prime 2} d x, \\
& \left.\quad\left|\sum_{j=1}^{l_{2}} b_{j}\left(q_{j}-1\right) \int_{\Omega}\right| u_{l \varepsilon}\right|^{q_{j}-2} u_{l \varepsilon}^{\prime} u_{l \varepsilon}^{\prime \prime} d x \mid \\
& \quad \leq \sum_{j=1}^{l_{2}} b_{i}\left(q_{i}-1\right) \kappa \int_{\Omega}\left(\left|u_{l \varepsilon}\right|^{q_{i}-2} u_{l \varepsilon}^{\prime \prime}\right)^{2} d x+\sum_{i=1}^{l_{2}} b_{j}\left(q_{j}-1\right) C_{1}(\kappa) \int_{\Omega} u_{l \varepsilon}^{\prime 2} d x
\end{aligned}
$$

for any $\kappa>0$. Let $\kappa$ be sufficiently small, we have the following inequality by use of Eqs. (12)-(15):

$$
\begin{aligned}
\frac{d}{d t} & {\left[\frac{1}{2} \int_{\Omega} u_{l \varepsilon}^{\prime \prime 2} d x+\frac{1}{\beta} \int_{\Omega}\left|\nabla u_{l \varepsilon}^{\prime}\right|^{\beta} d x+\frac{\varepsilon}{2} \int_{\Gamma_{1}} y_{l \varepsilon}^{\prime \prime 2} d \Gamma+\frac{1}{2} \int_{\Gamma_{1}} h q y_{l \varepsilon}^{\prime 2} d \Gamma\right]+\int_{\Gamma_{1}} h f y_{l \varepsilon}^{\prime \prime 2} d \Gamma } \\
& \leq C\left[\int_{\Omega}\left|\nabla u_{l \varepsilon}^{\prime}\right|^{2} d x+\int_{\Omega}\left|u_{l \varepsilon}^{\prime}\right|^{2} d x\right] .
\end{aligned}
$$


Integrating from 0 to $t$ and using Eq. (11), it follows that there exists a constant $M_{2}(T)>0$ such that

$$
\begin{aligned}
& \frac{1}{2} \int_{\Omega} u_{l \varepsilon}^{\prime \prime 2} d x+\frac{1}{\beta} \int_{\Omega}\left|\nabla u_{l \varepsilon}^{\prime}\right|^{\beta} d x+\frac{\varepsilon}{2} \int_{\Gamma_{1}} y_{l \varepsilon}^{\prime \prime 2} d \Gamma+\frac{1}{2} \int_{\Gamma_{1}} h q y_{l \varepsilon}^{\prime 2} d \Gamma+\int_{0}^{t} \int_{\Gamma_{1}} h f y_{l \varepsilon}^{\prime \prime 2} d \Gamma d \tau \\
& \quad \leq M_{2}(T) .
\end{aligned}
$$

Finally, combining with (10), (11), and (16) and using the Lions-Aubin theorem [29], we notice that $M_{1}(T)$ and $M_{2}(T)$ are independent of time, so we can obtain the existence of global solutions of Eqs. (1)-(2) by taking the limit of Eq. (7) with the similar method in [25], and the global solutions satisfy

$$
\begin{aligned}
& u \in L^{\infty}\left(0, T ; W^{1, m}(\Omega) \cap L^{q_{2}}(\Omega)\right), \quad u_{t} \in L^{\infty}\left(0, T ; W^{1, \beta}(\Omega)\right), \\
& \quad u_{t t} \in L^{\infty}\left(0, T ; L^{2}(\Omega)\right) \\
& \text { and } y, y_{t}, y_{t t} \in L^{\infty}\left(0, T ; L^{2}\left(\Gamma_{1}\right)\right) .
\end{aligned}
$$

Remark 2 The nonlinear term in Eq. (1) is more complicated than that of the viscoelastic wave equation in [6]. This paper uses different methods to deal with the nonlinear terms and Laplacian operators. And a similar method is used to solve the double dispersivedissipative wave equation with the Neumann condition in [27].

\section{Nonexistence theorem}

Let $H(t)=-E(t)$, using Eqs. (3) and (5), we have

$$
H(0) \leq H(t) \leq \sum_{i=1}^{l_{1}} \frac{a_{i}}{p_{i}} \int_{\Omega}|u|^{p_{i}} d x
$$

for any $t \geq 0$. Let

$$
L(t)=H^{(1-\sigma)}(t)+\varepsilon \int_{\Omega} u u_{t} d x-\frac{\varepsilon}{2} \int_{\Gamma_{1}} h(x) f(x) y^{2}(t) d \Gamma-\varepsilon \int_{\Gamma_{1}} h(x) u(t) y(t) d \Gamma,
$$

where $\varepsilon$ and $\sigma$ are constants.

Lemma 2 Suppose that $u(x, t)$ is a regular solution of Eq. (1) under condition (2), and the initial energy satisfies $E(0)<0$. If $\sigma<\min \left\{\frac{m-2}{p_{l_{1}}}, \frac{m-\beta}{p_{l_{1}}(\beta-1)}, 1\right\}$, then there exists a positive constant $r$ such that

$$
L^{\prime}(t) \geq r \varepsilon\left(H(t)+\|\nabla u\|_{m}^{m}+\left\|u_{t}\right\|_{2}^{2}+\int_{\Gamma_{1}} h(x) q(x) y^{2}(t) d \Gamma\right) .
$$

Proof Differentiating (18), the following equality can be obtained:

$$
\begin{aligned}
L^{\prime}(t)= & (1-\sigma) H^{-\sigma}(t) H^{\prime}(t)+\varepsilon \int_{\Omega} u_{t}^{2} d x+\varepsilon \int_{\Omega} u u_{t t} d x-\varepsilon \int_{\Gamma_{1}} h(x) f(x) y(t) y_{t}(t) d \Gamma \\
& -\varepsilon \int_{\Gamma_{1}} h(x) u_{t}(t) y(t) d \Gamma-\varepsilon \int_{\Gamma_{1}} h(x) u(t) y_{t}(t) d \Gamma .
\end{aligned}
$$


Using Eqs. (1)-(2) and Green's first formula, we obtain

$$
\begin{aligned}
& L^{\prime}(t)=(1-\sigma) H^{-\sigma}(t) H^{\prime}(t)+\varepsilon \int_{\Omega} u_{t}^{2} d x \\
& +\varepsilon \int_{\Omega} u\left[\alpha \operatorname{div}\left(|\nabla u|^{m-2} \nabla u\right)+\operatorname{div}\left(\left|\nabla u_{t}\right|^{\beta-2} \nabla u_{t}\right)+\gamma \Delta u_{t}\right. \\
& \left.+\sum_{i=1}^{l_{1}} a_{i}|u|^{p_{i}-2} u-\sum_{j=1}^{l_{2}} b_{j}|u|^{q_{j}-2} u\right] d x \\
& -\varepsilon \int_{\Gamma_{1}} h(x) f(x) y(t) y_{t}(t) d \Gamma-\varepsilon \int_{\Gamma_{1}} h(x) u_{t}(t) y(t) d \Gamma-\varepsilon \int_{\Gamma_{1}} h(x) u(t) y_{t}(t) d \Gamma \\
& =(1-\sigma) H^{-\sigma}(t) H^{\prime}(t)+\varepsilon \int_{\Omega} u_{t}^{2} d x \\
& -\varepsilon \alpha \int_{\Omega}|\nabla u|^{m} d x-\varepsilon \int_{\Omega}\left|\nabla u_{t}\right|^{\beta-2} \nabla u_{t} \nabla u d x-\varepsilon \gamma \int_{\Omega} \nabla u \nabla u_{t} d x \\
& +\varepsilon \sum_{i=1}^{l_{1}} a_{i} \int_{\Omega}|u|^{p_{i}} d x \\
& -\varepsilon \sum_{j=1}^{l_{2}} b_{j} \int_{\Omega}|u|^{q_{j}} d x+\varepsilon \int_{\Gamma_{1}}\left[\gamma \frac{\partial u_{t}}{\partial n}+\alpha|\nabla u|^{m-2} \frac{\partial u}{\partial n}+\left|\nabla u_{t}\right|^{\beta-2} \frac{\partial u_{t}}{\partial n}\right] u d \Gamma \\
& -\varepsilon \int_{\Gamma_{1}} h(x) f(x) y(t) y_{t}(t) d \Gamma-\varepsilon \int_{\Gamma_{1}} h(x) u_{t}(t) y(t) d \Gamma-\varepsilon \int_{\Gamma_{1}} h(x) u(t) y_{t}(t) d \Gamma \\
& =(1-\sigma) H^{-\sigma}(t) H^{\prime}(t)+\varepsilon \int_{\Omega} u_{t}^{2} d x \\
& -\varepsilon \alpha \int_{\Omega}|\nabla u|^{m} d x-\varepsilon \int_{\Omega}\left|\nabla u_{t}\right|^{\beta-2} \nabla u_{t} \nabla u d x \\
& -\varepsilon \gamma \int_{\Omega} \nabla u \nabla u_{t} d x+\varepsilon \sum_{i=1}^{l_{1}} a_{i} \int_{\Omega}|u|^{p_{i}} d x \\
& -\varepsilon \sum_{j=1}^{l_{2}} b_{j} \int_{\Omega}|u|^{q_{j}} d x+\varepsilon \int_{\Gamma_{1}} h(x) q(x) y^{2}(t) d \Gamma .
\end{aligned}
$$

We can obtain the following inequalities from Young's inequality and Hölder's inequality:

$$
\begin{aligned}
& \int_{\Omega} \nabla u \nabla u_{t} d x \leq \frac{1}{4 \kappa} \int_{\Omega}|\nabla u|^{2} d x+\kappa \int_{\Omega}\left|\nabla u_{t}\right|^{2} d x \\
& \int_{\Omega}\left|\nabla u_{t}\right|^{\beta-2} \nabla u_{t} \nabla u d x \leq \frac{\delta^{\beta}}{\beta} \int_{\Omega}|\nabla u|^{\beta} d x+\frac{\beta-1}{\beta} \delta^{-\frac{\beta}{\beta-1}} \int_{\Omega}\left|\nabla u_{t}\right|^{\beta} d x .
\end{aligned}
$$

Hence,

$$
\begin{aligned}
L^{\prime}(t) \geq & (1-\sigma) H^{-\sigma}(t) H^{\prime}(t)+\varepsilon \int_{\Omega} u_{t}^{2} d x-\varepsilon \alpha \int_{\Omega}|\nabla u|^{m} d x \\
& -\varepsilon \frac{\delta^{\beta}}{\beta} \int_{\Omega}|\nabla u|^{\beta} d x-\varepsilon \frac{\beta-1}{\beta} \delta^{-\frac{\beta}{\beta-1}} \int_{\Omega}\left|\nabla u_{t}\right|^{\beta} d x
\end{aligned}
$$




$$
\begin{aligned}
& -\frac{\varepsilon \gamma}{4 \kappa} \int_{\Omega}|\nabla u|^{2} d x-\varepsilon \gamma \kappa \int_{\Omega}\left|\nabla u_{t}\right|^{2} d x+\varepsilon \sum_{i=1}^{l_{1}} a_{i} \int_{\Omega}|u|^{p_{i}} d x \\
& -\varepsilon \sum_{j=1}^{l_{2}} b_{j} \int_{\Omega}|u|^{q_{j}} d x+\varepsilon \int_{\Gamma_{1}} h(x) q(x) y^{2}(t) d \Gamma .
\end{aligned}
$$

Taking $\kappa=M_{1} H^{-\sigma}(t)$ and $\delta^{-\frac{\beta}{\beta-1}}=M_{2} H^{-\sigma}(t)$, and using $f(x), h(x), H(t)>0$, we obtain

$$
\begin{aligned}
L^{\prime}(t) \geq & (1-\sigma) H^{-\sigma}(t) H^{\prime}(t)+\varepsilon \int_{\Omega} u_{t}^{2} d x-\varepsilon \alpha \int_{\Omega}|\nabla u|^{m} d x-\frac{\varepsilon \gamma}{4 M_{1}} H^{\sigma}(t) \int_{\Omega}|\nabla u|^{2} d x \\
& -\varepsilon \gamma M_{1} H^{-\sigma}(t) \int_{\Omega}\left|\nabla u_{t}\right|^{2} d x-\frac{\varepsilon M_{2}^{-(\beta-1)}}{\beta} H^{\sigma(\beta-1)}(t) \int_{\Omega}|\nabla u|^{\beta} d x \\
& -\varepsilon \frac{\beta-1}{\beta} M_{2} H^{-\sigma}(t) \int_{\Omega}\left|\nabla u_{t}\right|^{\beta} d x \\
& +\varepsilon \sum_{i=1}^{l_{1}} a_{i} \int_{\Omega}|u|^{p_{i}} d x-\varepsilon \sum_{j=1}^{l_{2}} b_{j} \int_{\Omega}|u|^{q_{j}} d x+\varepsilon \int_{\Gamma_{1}} h(x) q(x) y^{2}(t) d \Gamma .
\end{aligned}
$$

Let $M=\gamma M_{1}+\frac{\beta-1}{\beta} M_{2}$, using the energy functional (4), we have

$$
\begin{aligned}
L^{\prime}(t) \geq & (1-\sigma-\varepsilon M) H^{-\sigma}(t) H^{\prime}(t)+\varepsilon \int_{\Omega} u_{t}^{2} d x-\varepsilon \alpha \int_{\Omega}|\nabla u|^{m} d x \\
& -\frac{\varepsilon \gamma}{4 M_{1}} H^{\sigma}(t) \int_{\Omega}|\nabla u|^{2} d x \\
& -\frac{\varepsilon M_{2}^{-(\beta-1)}}{\beta} H^{\sigma(\beta-1)}(t) \int_{\Omega}|\nabla u|^{\beta} d x++\varepsilon \sum_{i=1}^{l_{1}} a_{i} \int_{\Omega}|u|^{p_{i}} d x-\varepsilon \sum_{j=1}^{l_{2}} b_{j} \int_{\Omega}|u|^{q_{j}} d x \\
& +\varepsilon \int_{\Gamma_{1}} h(x) q(x) y^{2}(t) d \Gamma+\varepsilon M H^{-\sigma}(t) \int_{\Gamma_{1}} h(x) f(x) y_{t}^{2}(t) d \Gamma \\
\geq & (1-\sigma-\varepsilon M) H^{-\sigma}(t) H^{\prime}(t)+\varepsilon \int_{\Omega} u_{t}^{2} d x-\varepsilon \alpha \int_{\Omega}|\nabla u|^{m} d x \\
& -\frac{\varepsilon \gamma}{4 M_{1}} H^{\sigma}(t) \int_{\Omega}|\nabla u|^{2} d x \\
& -\frac{\varepsilon M_{2}^{-(\beta-1)}}{\beta} H^{\sigma(\beta-1)}(t) \int_{\Omega}|\nabla u|^{\beta} d x+\varepsilon \sum_{i=1}^{l_{1}} a_{i} \int_{\Omega}|u|^{p_{i}} d x \\
& -\varepsilon \sum_{j=1}^{l_{2}} b_{j} \int_{\Omega}|u|^{q_{j}} d x+\varepsilon \int_{\Gamma_{1}} h(x) q(x) y^{2}(t) d \Gamma .
\end{aligned}
$$

From the definition of $H(t)$, it follows that there is a constant $k$ such that

$$
\begin{aligned}
L^{\prime}(t) \geq & (1-\sigma-\varepsilon M) H^{-\sigma}(t) H^{\prime}(t)+k H(t)+\left(\varepsilon+\frac{k}{2}\right) \int_{\Omega} u_{t}^{2} d x \\
& +\left(\frac{k}{m}-\varepsilon\right) \alpha \int_{\Omega}|\nabla u|^{m} d x
\end{aligned}
$$




$$
\begin{aligned}
& +\sum_{i=1}^{l_{1}}\left(\varepsilon-\frac{k}{p_{i}}\right) a_{i} \int_{\Omega}|u|^{p_{i}} d x+\sum_{j=1}^{l_{2}}\left(\frac{k}{q_{j}}-\varepsilon\right) b_{j} \int_{\Omega}|u|^{q} d x \\
& +\left(\frac{k}{2}+\varepsilon\right) \int_{\Gamma_{1}} h(x) q(x) y^{2}(t) d \Gamma \\
& -\frac{\varepsilon}{\beta} M_{2}^{-(\beta-1)} H^{\sigma(\beta-1)}(t) \int_{\Omega}|\nabla u|^{\beta} d x-\frac{\varepsilon \gamma}{4 M_{1}} H^{\sigma}(t) \int_{\Omega}|\nabla u|^{2} d x .
\end{aligned}
$$

Using $W^{1, m}(\Omega) \rightarrow H^{1}(\Omega), W^{1, m}(\Omega) \rightarrow L^{p_{i}}(\Omega), m \geq \beta$, and Inequality (17), after simple calculation, it can be concluded

$$
\begin{aligned}
H^{\sigma}(t) \int_{\Omega}|\nabla u|^{2} d x & \leq C \sum_{i=1}^{l_{1}}\left(\frac{a}{p_{i}}\right)^{\sigma}\left(\int_{\Omega}|u|^{p_{i}} d x\right)^{\sigma} \int_{\Omega}|\nabla u|^{2} d x \\
& \leq C \sum_{i=1}^{l_{1}}\left(\frac{a}{p_{i}}\right)^{\sigma}\left(\int_{\Omega}|\nabla u|^{m} d x\right)^{\frac{\sigma p_{i}+2}{m}}
\end{aligned}
$$

and

$$
H^{\sigma(\beta-1)}(t) \int_{\Omega}|\nabla u|^{\beta} d x \leq C \sum_{i=1}^{l_{1}}\left(\frac{a_{i}}{p_{i}}\right)^{\sigma(\beta-1)}\left(\int_{\Omega}|\nabla u|^{m} d x\right)^{\frac{p_{i} \sigma(\beta-1)+\beta}{m}} .
$$

For any constants $z \geq 0$ and $M>0$, the algebraic inequality

$$
z^{\nu} \leq z+1 \leq\left(1+\frac{1}{M}\right)(z+M), \quad(0<v<1)
$$

holds. Using the known condition $\sigma<\min \left\{\frac{m-2}{p_{l_{1}}}, \frac{m-\beta}{p_{l_{1}}(\beta-1)}\right\}$, we have $\sigma<\frac{m-2}{p_{i}}$ and $\sigma<\frac{m-\beta}{p_{i}(\beta-1)}$ for any $1 \leq i \leq l_{1}$. Hence from (27) the following inequalities can be acquired:

$$
\begin{aligned}
\left(\int_{\Omega}|\nabla u|^{m} d x\right)^{\frac{\sigma p_{i}+2}{m}} & \leq\left(1+\frac{1}{H(0)}\right)\left(\int_{\Omega}|\nabla u|^{m} d x+H(0)\right) \\
& \leq\left(1+\frac{1}{H(0)}\right)\left(\int_{\Omega}|\nabla u|^{m} d x+H(t)\right)
\end{aligned}
$$

and

$$
\left(\int_{\Omega}|\nabla u|^{m} d x\right)^{\frac{p_{i} \sigma(\beta-1)+\beta}{m}} \leq\left(1+\frac{1}{H(0)}\right)\left(\int_{\Omega}|\nabla u|^{m} d x+H(t)\right) .
$$

From Inequalities (25) and (26), we can obtain that there exist two positive constants $N_{1}$ and $N_{2}$ such that

$$
\frac{\varepsilon \gamma}{4 M_{1}} H^{\sigma}(t) \int_{\Omega}|\nabla u|^{2} d x \leq \varepsilon N_{1}\left(\int_{\Omega}|\nabla u|^{m} d x+H(t)\right)
$$

and

$$
\frac{\varepsilon}{\beta} M_{2}^{-(\beta-1)} H^{\sigma(\beta-1)}(t) \int_{\Omega}|\nabla u|^{\beta} d x \leq \varepsilon N_{2}\left(\int_{\Omega}|\nabla u|^{m} d x+H(t)\right) .
$$


Consequently, taking $k=\frac{1}{2}\left(N_{1}+N_{2}+\frac{m N_{1}}{\alpha}+\frac{m N_{2}}{\alpha}+m+p_{1}+q_{1}\right) \varepsilon$, we obtain from (24) that there exists a positive constant $r$ such that

$$
\begin{aligned}
L^{\prime}(t) \geq & (1-\sigma-\varepsilon M) H^{-\sigma}(t) H^{\prime}(t)+\left(k-N_{1} \varepsilon-N_{2} \varepsilon\right) H(t)+\left(\varepsilon+\frac{k}{2}\right) \int_{\Omega} u_{t}^{2} d x \\
& +\left(\frac{k}{m}-\varepsilon-\frac{N_{1}}{\alpha} \varepsilon-\frac{N_{2}}{\alpha} \varepsilon\right) \alpha \int_{\Omega}|\nabla u|^{m} d x+\sum_{i=1}^{l_{1}}\left(\varepsilon-\frac{k}{p_{i}}\right) a_{i} \int_{\Omega}|u|^{p_{i}} d x \\
& +\sum_{j=1}^{l_{2}}\left(\frac{k}{q_{j}}-\varepsilon\right) b_{j} \int_{\Omega}|u|^{q} d x+\left(\frac{k}{2}+\varepsilon\right) \int_{\Gamma_{1}} h(x) q(x) y^{2}(t) d \Gamma \\
\geq & (1-\sigma-\varepsilon M) H^{-\sigma}(t) H^{\prime}(t) \\
& +r \varepsilon\left[H(t)+\int_{\Omega} u_{t}^{2} d x+\int_{\Omega}|\nabla u|^{m} d x+\int_{\Gamma_{1}} h(x) q(x) y^{2}(t) d \Gamma\right] .
\end{aligned}
$$

Taking $0<\varepsilon<\frac{1-\sigma}{M}$, we can get the following formula from (18):

$$
L(0)=H^{(1-\sigma)}(0)+\varepsilon \int_{\Omega} u_{0}(x) u_{1}(x) d x-\frac{\varepsilon}{2} \int_{\Gamma_{1}} h(x) f(x) y^{2}(0) d \Gamma-\varepsilon \int_{\Gamma_{1}} h(x) u(0) y(0) d \Gamma
$$

$>0$.

Using Inequality (32), it holds

$$
L^{\prime}(t) \geq r \varepsilon\left[H(t)+\int_{\Omega} u_{t}^{2} d x+\int_{\Omega}|\nabla u|^{m} d x+\int_{\Gamma_{1}} h(x) q(x) y^{2}(t) d \Gamma\right] .
$$

After integral, we can get $L(t) \geq L(0)>0,(\forall t \geq 0)$.

Theory 2 Suppose $\alpha, \gamma, a_{i}, b_{j}>0$ and $2<\beta<m<q_{l_{2}}<\cdots<q_{2}<q_{1}<p_{1}<p_{2}<\cdots<p_{l_{1}}<$ $r^{*}$, where $r^{*}$ is the critical Sobolev index in $W^{1, m}(\Omega)$. If the initial energy $E(0)<0$, then any regular solutions of Eqs. (1)-(2) must blow up in finite time.

Proof Firstly, it is proved that when $0<\sigma<\frac{m-2}{2 m}$, there exists a positive constant $C$ such that

$$
L^{\frac{1}{1-\sigma}}(t) \leq C\left(H(t)+\int_{\Omega}|\nabla u|^{m} d x+\int_{\Omega}\left|u_{t}\right|^{2} d x+\int_{\Gamma_{1}} h(x) q(x) y^{2}(t) d \Gamma\right) .
$$

In fact, using (18) and $f, h>0$, we get

$$
L^{\frac{1}{1-\sigma}}(t) \leq C(\varepsilon, \sigma)\left[H(t)+\left(\int_{\Omega} u u_{t} d x\right)^{\frac{1}{1-\sigma}}+\left(\int_{\Gamma_{1}} h(x) u(t) y(t) d \Gamma\right)^{\frac{1}{1-\sigma}}\right] .
$$


Furthermore, by using Hölder's inequality and Young's inequality, we can get

$$
\begin{aligned}
\left(\int_{\Omega} u u_{t} d x\right)^{\frac{1}{1-\sigma}} & \leq\left(\int_{\Omega} u^{2} d x\right)^{\frac{1}{2(1-\sigma)}}\left(\int_{\Omega} u_{t}^{2} d x\right)^{\frac{1}{2(1-\sigma)}} \\
& \leq C\left(\int_{\Omega}|u|^{m} d x\right)^{\frac{1}{m(1-\sigma)}}\left(\int_{\Omega} u_{t}^{2} d x\right)^{\frac{1}{2(1-\sigma)}} \\
& \leq C\left[\left(\int_{\Omega}|u|^{m} d x\right)^{\frac{\mu}{m(1-\sigma)}}+\left(\int_{\Omega} u_{t}^{2} d x\right)^{\frac{\theta}{2(1-\sigma)}}\right]
\end{aligned}
$$

where $\frac{1}{\mu}+\frac{1}{\theta}=1$. Taking $\theta=2(1-\sigma)$, we have $\mu=\frac{2(1-\sigma)}{1-2 \sigma}$. By Poincare's inequality, it follows

$$
\left(\int_{\Omega} u u_{t} d x\right)^{\frac{1}{1-\sigma}} \leq C\left[\left(\int_{\Omega}|\nabla u|^{m} d x\right)^{\frac{2}{m(1-2 \sigma)}}+\int_{\Omega} u_{t}^{2} d x\right] .
$$

If $0<\sigma<\frac{m-2}{2 m}$, then we get $0<\frac{2}{m(1-2 \sigma)}<1$. From (17), (27), and (35), we obtain

$$
\begin{aligned}
\left(\int_{\Omega} u u_{t} d x\right)^{\frac{1}{1-\sigma}} & \leq C\left(\int_{\Omega}|\nabla u|^{m} d x+H(0)+\int_{\Omega} u_{t}^{2} d x\right) \\
& \leq C\left(\int_{\Omega}|\nabla u|^{m} d x+H(t)+\int_{\Omega} u_{t}^{2} d x\right) .
\end{aligned}
$$

On the other hand, by Hölder's inequality and $W_{0}^{1, m}(\Omega) \rightarrow L^{2}\left(\Gamma_{1}\right)$, we have

$$
\begin{aligned}
\int_{\Gamma_{1}} h(x) u(t) y(t) d \Gamma & =\left|\int_{\Gamma_{1}} \frac{h(x) q(x)}{q(x)} u(t) y(t) d \Gamma\right| \\
& \leq \frac{\|h\|_{\infty}^{\frac{1}{2}}\|q\|_{\infty}^{\frac{1}{2}}}{q_{0}}\left(\int_{\Gamma_{1}} h(x) q(x) y^{2}(t) d \Gamma\right)^{\frac{1}{2}}\left(\int_{\Gamma_{1}} u^{2}(t) d \Gamma\right)^{\frac{1}{2}} \\
& \leq C\left(\int_{\Gamma_{1}} h(x) q(x) y^{2}(t) d \Gamma\right)^{\frac{1}{2}}\left(\int_{\Omega}|\nabla u|^{m} d x\right)^{\frac{1}{m}}
\end{aligned}
$$

Hence, using (27) and the similar method above, it can be obtained

$$
\begin{aligned}
\left(\int_{\Gamma_{1}} h(x) u(t) y(t) d \Gamma\right)^{\frac{1}{1-\sigma}} & \leq C\left(\int_{\Gamma_{1}} h(x) q(x) y^{2}(t) d \Gamma\right)^{\frac{1}{2(1-\sigma)}}\left(\int_{\Omega}|\nabla u|^{m} d x\right)^{\frac{1}{m(1-\sigma)}} \\
& \leq C\left[\int_{\Gamma_{1}} h(x) q(x) y^{2}(t) d \Gamma+\left(\int_{\Omega}|\nabla u|^{m} d x\right)^{\frac{2}{m(1-2 \sigma)}}\right] \\
& \leq C\left(\int_{\Omega}|\nabla u|^{m} d x+H(t)+\int_{\Gamma_{1}} h(x) q(x) y^{2}(t) d \Gamma\right) .
\end{aligned}
$$

From (34), (36), and (37), we get

$$
L^{\frac{1}{1-\sigma}}(t) \leq C\left(H(t)+\int_{\Omega}|\nabla u|^{m} d x+\int_{\Omega}\left|u_{t}\right|^{2} d x+\int_{\Gamma_{1}} h(x) q(x) y^{2}(t) d \Gamma\right),
$$

where $C$ is only related to $\sigma, \varepsilon, q_{0},\|q\|_{\infty},\|h\|_{\infty}$, and $H(0)$. 
Taking $\sigma<\min \left\{\frac{m-2}{p_{l_{1}}}, \frac{m-\beta}{p_{l_{1}}(\beta-1)}, \frac{m-2}{2 m}, 1\right\}$, by Inequalities (33) and (19) in Lemma 2, it follows that there exists a constant $\xi>0$ such that

$$
L^{\prime}(t) \geq \xi L^{\frac{1}{1-\sigma}}(t)
$$

for any $t \geq 0$. Integrating the above formula with respect to $t$ on $[0, t]$, we get

$$
L^{\frac{\sigma}{1-\sigma}}(t) \geq \frac{1}{L^{\frac{-\sigma}{1-\sigma}}(0)-\frac{\sigma \xi}{1-\sigma} t}
$$

for any $t \geq 0$. Hence there exists $T^{*} \leq \frac{1-\sigma}{\sigma \xi L \frac{\sigma}{1-\sigma}(0)}$ such that $\lim _{t \rightarrow T^{*}} L(t)=\infty$, that means the regular solution $u(x, t)$ must blow up in finite time.

Remark 3 Compared with the quasilinear viscoelastic wave equation in [8] and [9], we not only prove the existence of global solutions of the equation, but also give the more complex nonlinear terms.

\section{Conclusion}

When the wave equation has nonlinear source terms, $m$ and $\beta$ order Laplacian operators, we discuss the initial boundary problem of the wave equation under acoustic boundary conditions. We firstly prove that the energy corresponding to any regular solutions is nonincreasing with respect to $t$. Combined with the theory of convergence, the existence of the global solution is proved by using the approximate solutions constructed by the FaedoGalerkin method. On the other hand, we investigate the nonexistence of global solutions and give the sufficient conditions of blow-up of any regular solutions in finite time.

\section{Acknowledgements}

The authors are thankful to the honorable reviewers and editors for their valuable reviewing of the manuscript.

\section{Funding}

The work was supported by the Key Projects of Natural Science Research in Colleges and Universities of Anhui Province (KJ2019A0666, KJ2020A0735),the Key Scientific Research Projects of Suzhou University (2020yzd06), the Scientific Research Platform Projects of Suzhou University (2020ykf17), the Research Projects of Anhui Education Department (2020szsfkc0998).

Availability of data and materials

Data sharing not applicable to this article as no datasets were generated or analyzed during the current study.

Competing interests

The authors declare that they have no competing interests.

Authors' contributions

The authors declare that the study was realized in collaboration with the same responsibility. All authors read and approved the manuscript.

Author details

'School of Mathematics and Statistics, Suzhou University, Suzhou, 234000, China. ${ }^{2}$ Medical College, Henan University, Kaifeng, 475001, China.

\section{Publisher's Note}

Springer Nature remains neutral with regard to jurisdictional claims in published maps and institutional affiliations. 


\section{References}

1. Morse, P.M., Ingard, K.V.: Theoretical Acoustics. McGraw-Hill, New York (1968)

2. Beale, J.T., Rosencrans, S.I: Acoustic boundary conditions. Bull. Am. Math. Soc. 80, 1276-1278 (1974)

3. Gerbi, S., Said-Houari, B.: Local existence and exponential growth for a semilinear damped wave equation with dynamical boundary conditions. Adv. Differ. Equ. 13, 1051-1074 (2008)

4. Frigeri, S.: Attractor for the semilinear damped wave equations with an acoustic boundary condition. J. Evol. Equ. 10, 29-58 (2010)

5. Liu, W.J.: Arbitrary rate of decay for a viscoelastic equation with acoustic boundary conditions. Appl. Math. Lett. 38, 155-161 (2014)

6. Yu, J.L., Shang, Y.D., Di, H.F: Global existence, nonexistence, and decay of solutions for a viscoelastic wave equation with nonlinear boundary damping and source terms. J. Math. Phys. 61, 071503 (2020)

7. $\mathrm{Mi}$, J.L., Jong, Y.P.: Energy decay of solutions of nonlinear viscoelastic problem with the dynamic and acoustic boundary conditions. Bound. Value Probl. 1, 1-26 (2018)

8. Kang, Y.H., Park, J.Y., Park, S.H.: A global nonexistence of solutions for a quasilinear viscoelastic wave equation with acoustic boundary conditions. Bound. Value Probl. 139, 1-19 (2018)

9. Jin, M.J., Jong, Y.P., Yong, H.K.: Global nonexistence of solutions for a quasilinear wave equation with acoustic boundary conditions. Bound. Value Probl. 42, 1-10 (2017)

10. Jin, M.J., Jong, Y.P., Yong, H.K.: Global nonexistence of solutions for a nonlinear wave equation with time delay and acoustic boundary conditions. Comput. Math. Appl. 76(31), 661-671 (2018)

11. Jin, M.J., Jong, Y.P., Yong, H.K.: Energy decay rates for the semilinear wave equation with memory boundary condition and acoustic boundary conditions. Comput. Math. Appl. 73(9), 1975-1986 (2017)

12. Tae, G.H.: General decay estimates for the wave equation with acoustic boundary conditions in domains with nonlocally reacting boundary. Appl. Math. Lett. 60, 43-49 (2016)

13. Tae, G.H.: Energy decay for the wave equation of variable coefficients with acoustic boundary conditions in domains with nonlocally reacting boundary. Appl. Math. Lett. 76, 201-207 (2018)

14. Sattinger, D.H.: On global solution of nonlinear hyperbolic equations. Arch. Ration. Mech. Anal. 30, 148-172 (1968)

15. Payne, L.E., Sattinger, D.H.: Sadle points and instability of nonlinear wave equations. Isr. J. Math. 22, 273-303 (1975)

16. Liu, Y.C.: On potential wells and vacuum isolating of solutions for semilinear wave equations. J. Differ. Equ. 192, 155-169 (2003)

17. Xu, R.Z., Shen, J.H., Liu, Y.C.: Potential well and its applications to wave equations with source terms of different signs. Chin. J. Eng. Math. 24(5), 931-934 (2007)

18. Xu, R.Z., Yu, T.: Remarks on wave equations involving two opposite nonlinear source terms. J. Appl. Math. Comput. 29 $15-18(2009)$

19. Liu, Y.C., Xu, R.Z., Yu, T.: Wave equations with several nonlinear source terms. Appl. Math. Mech. 28(9), 1079-1086 (2007)

20. Xu, R.Z., Xu, C.: Sharp conditions of global existence for second-order derivative nonlinear Schrödinger equations with combined power-type nonlinearities. J. Appl. Math. Mech. 93(1), 29-37 (2013)

21. Xu, R.Z.: Cauchy problem of generalized Boussinesq equation with combined power-type nonlinearities. Math. Methods Appl. Sci. 34(18), 2318-2328 (2011)

22. Jin, S.B., Zhang, Z.F., Zhao, M.L.: A class of wave equation with nonlinear damping and multiple terms. J. Wuhan Univ. Natur. Sci. Ed. 62(5), 464-470 (2016)

23. Xu, R.Z., Wang, X.H., Xu, H.C., Zhang, M.Y.: Arbitrary energy global existence for wave equation with combined power-type nonlinearities of different signs. Bound. Value Probl. 1, 214 (2016)

24. Lian, W., Xu, R.Z:: Global well-posedness of nonlinear wave equation with weak and strong damping terms and logarithmic source term. Adv. Nonlinear Anal. 9(1), 613-632 (2020)

25. Tae, G.H., Jong, Y.P.: Existence of solutions for the Kirchhoff-type wave equation with memory term and acoustic boundary conditions. Numer. Funct. Anal. Optim. 31(8), 921-935 (2010)

26. Liu, W.J., Chen, K.W.: Existence and general decay for nondissipative distributed systems with boundary frictional and memory dampings and acoustics boundary conditions. Z. Angew. Math. Phys. 66, 1595-1614 (2015)

27. Di, H.F., Shang, Y.D.: Global existence and asymptotic behavior of solutions for the double dispersive-dissipative wave equation with nonlinear damping and source terms. Bound. Value Probl. 29, 1-15 (2015)

28. Messaoudi, S.A.: Blow-up of positive-initial-energy solutions of a nonlinear viscoelastic hyperbolic equation. J. Math. Anal. Appl. 320, 902-915 (2006)

29. Lions, J.L.: Quelques Méthodes de Résolution des Problèmes aux Limites Non Linéaires. Dunod, Paris (1969)

\section{Submit your manuscript to a SpringerOpen ${ }^{\circ}$ journal and benefit from:}

- Convenient online submission

Rigorous peer review

- Open access: articles freely available online

- High visibility within the field

Retaining the copyright to your article

Submit your next manuscript at $\gg$ springeropen.com 